\title{
Rate of infant carrying impacts infant spontaneous motor tempo
}

\author{
Sinead Rocha \\ University of Cambridge
}

\author{
Victoria Southgate \\ University of Copenhagen
}

\author{
Denis Mareschal \\ Birkbeck, University of London
}

\begin{abstract}
Rhythm production is a critical component of human interaction, not least forming the basis of our musicality. Infants demonstrate a Spontaneous Motor Tempo (SMT), or natural rate of rhythmic movement. Here we ask whether infant SMT is influenced by the rate of locomotion infants experience when being carried. Ten-month-old, non-walking infants were tested using a free drumming procedure before and after ten minutes of being carried by an experimenter walking at a slower (98 BPM) or faster (138 BPM) than average tempo. We find that infant SMT is differentially impacted by carrying experience dependent on the tempo at which they were carried: Infants in the slow-walked group exhibited a slower SMT from pre-test to post-test, whilst infants in the fast-walked group showed a faster SMT from pre-test to post-test. Heart rate data suggest that this effect is not due to a general change in state of arousal. We argue that being carried during caregiver locomotion is a predominant experience for infants throughout the first years of life, and as a source of regular, vestibular, information, may at least partially form the basis of their sense of rhythm.
\end{abstract}

Keywords: Rhythm, Spontaneous Motor Tempo, Infants, Locomotion, Development

One of the most intriguing aspects of music is that it makes us want to move (Janata, Tomic \& Haberman, 2012). The bidirectional relationship between auditory rhythms and movement has been well documented (Phillips-Silver \& Trainor, 2005; 2008; Trainor et al., 2009). The underlying quasiperiodic pulse, or tactus, of music, is the simplest form of rhythm. In adult humans, it is this beat that we tap along to when listening to music, defining the tempi of our movement. The ability to synchronise one's movement to the beat develops slowly across childhood (see Hannon, Nave-Blodgett \& Nave, 2018), and yet, newborns are able to detect the beat in music (Winkler et al., 2009), and infants spend up to $40 \%$ of their time in rhythmic movement (Thelen, 1979; 1981). Understanding the ontogeny of rhythm can offer insight into the experiences that provide the earliest building blocks of music. The current paper is concerned with the prevalent behaviour of infant carrying, and how experience of locomotion, even before locomoting oneself, may influence the tempo of spontaneous rhythmic movement.

Trevarthen (2000) argues that human bipedal locomotion provides us with a rhythm, with regular footfalls forming an underlying beat to daily life, but combined with likely quasi-independent movements of the upper body and limbs, providing the possibility of a multiplicity of rhythms all at once. In studies of natural movement to music, there is evidence that different body parts group into different eigenmovements, such that the beat level of music tends to be represented over the body in vertical actions, especially of the torso, compared to the meter level, often embodied as mediolateral movement over the arms or body sway (Toiviainen, Luck \& Thompson, 2010; Burger et al., 2014). The regular vertical 'bounce' of stepping may feed our rhythmic preferences. It has long been proposed that the core of the sensory-motor relationship ubiquitous in rhythm may be primarily vestibular in nature (Todd, O'Boyle \& Lee, 1999; Todd \& Lee, 2015).

The tempo of most music and human locomotion coincide (Mishima, 1965; Ayres, 1973; Fraisse, 1982; Styns et al., 2007; Trainor, 2007). It is possible that this relationship is causal, such that the experience of rhythmic walking forms our natural rate of rhythmic movement, termed Spontaneous Motor Tempo (SMT; Fraisse 1982). The rate at which an individual walks is in part determined by the length of their limbs and other such anthropometric features, due to the pendulum nature of the leg swing and aerobic cost optimisation that depends on stature (e.g. Bertram, 2005). Anthropometrics correlate with one's SMT, as measured by finger tapping (Mishima, 1965), and full body dancing (Dahl et al., 2014), as well as preferred tempo in a perceptual listening task (Todd, Cousins \& Lee, 2007). Further, data suggest that infant SMT is predicted by parent anthropometrics, such that infants of taller parents have a slower SMT (Rocha, Southgate \& Mareschal, 2021). Infants provide an interesting test case: Whilst a correlation between own body size and natural rate of movement could be explained as the product of biomechanical res- 
onance, or a natural frequency of movement across the body, the existence of a relationship between parent body size and infant SMT supports the idea that experience of locomotion - in this case when carried by the caregiver - drives our basic rhythmic preferences. Prenatally, infants can hear the mother's footfall as well as experience the corresponding movement from within the womb; Teie (2016) suggests a direct mapping of prenatal experience of the mother's walking to the musical property of 'pulse', or beat. Then, for a protracted period of postnatal development, infants cannot walk independently. Accordingly, in contemporary hunter-gatherer societies, infants are held or carried for most of the day (Lozoff, Brittenham \& Klaus, 1978). The fetus and newborn responds to vestibular-tactile-somatosensory rhythms, (see review by Provasi et al., 2014), and vestibular information is known to impact rhythm perception from infancy (Phillips-Silver and Trainor, 2005, 2008; Trainor et al., 2009). In a different approach, Ayres (1973) analysed data collected from 54 traditional, historically independent societies, and found that societies where infants are carried, either in a sling or on the body without support, produce a higher percentage of music with a regular rhythm than societies that use a cradle, cradleboard or hammock. Ayres (1973) concluded that societal preference for a regular rhythm is correlated with the nature and frequency of infant carrying in that population.

Rocha et al. (2021) developed a simple free drumming task that measured the SMT of infants across the first years of life, and found that infant SMT was predicted by parental body size, but not infant's own body size. Specifically, parent height predicted infant SMT such that infants with taller parents showed a slower SMT than infants with shorter parents. Whilst the dependent variables in Rocha et al. (2021) were anthropometric indices, results were interpreted as indicative of the vast amounts of information gained by infants when they are carried by their caregiver, at the caregiver's walking cadence.

To date it has not been directly tested whether the experience of being carried during locomotion impacts infant rhythm production, though seminal work shows even two minutes of vestibular movement can bias rhythm perception (Phillips-Silver \& Trainor, 2005). The current study aims to experimentally manipulate infant SMT with a short lab based carrying intervention, employing a pre-test, training, post-test design. Infant SMT was measured at pre- and post-test using the free drumming task employed in Rocha et al. (2021). During the training period, non-walking, ten month-old infants were carried in a forward facing baby carrier and walked by the experimenter at either a Fast (138 BPM) or Slow (98 BPM) pace, for ten minutes. The Fast and Slow rates reflect the extreme values of the normal range of cadence in free-speed walking for females aged 18-49 years (Whittle, 1990). Carrying rates were chosen to give the infants experience that was likely novel, so far as not shadowing population mean cadence, but still ecological, and not a non-naturalistic pace.

To further knowledge of the relationship between body size and rhythm, anthropometrics, including height and weight, of both infant and parent were taken. Additionally, parent SMT was measured via free drumming, free tapping and a free treadmill walking measure. In line with Rocha et al. (2021), we predict that parental body size will predict infant SMT, such that infants with a larger parent will drum more slowly at pre-test than infants with a smaller parent. We further hypothesise that parent body size will predict parent's own SMT and walking cadence.

Our primary hypothesis centers on the experimental manipulation, and predicts that infants in the Fast condition will drum faster at post-test than pre-test, and that infants in the Slow condition will drum more slowly at post-test than pre-test. Finally, to ensure that changes in SMT in the current study are not the product of a general state of higher or lower arousal, we measure infant heart rate whilst at rest, immediately before and after carrying, and predict no change from pre- to post-test.

\section{Participants}

\section{Method}

Forty-four ten-month-olds took part in the study, in a between-subjects design ( 22 female; mean age $=10$ months $(\mathrm{M}=305$ days, range $=290$ to 332 days $)$ ). Twenty-three infants $(\mathrm{M}=304$ days, range $=291$ to 323 days)) were randomly allocated to the Fast condition, and 21 infants ( $\mathrm{M}=305$ days, range $=290$ to 332 days) to the Slow condition. Infants had to provide at least one bout of drumming at both pre- and post-test for inclusion in the intervention analyses. 15 infants did not do so, which is in line with the attrition rate of infant studies (Stets, Stahl \& Reid, 2012). A further two infants were excluded for sporadic, arhythmic, drumming, using the exclusion criteria set in Rocha et al. (2021), further detail below. The intervention analysis therefore composed of 14 infants in the Fast condition ( $\mathrm{M}=304$ days, range = 291 to 322 days) and 13 infants in the Slow condition (M $=305$ days, range $=291$ to 332 days).

In the analyses of anthropometric/questionnaire data, further infants/caregivers who provided valid data for each measure were included, corresponding degrees of freedom are provided. Only non-walking infants were 
recruited for the study. All caregivers gave written, informed consent concerning the experimental procedure for themself and their infant. Infants received a certificate and a t-shirt as a thank you for participation.

\section{Procedure}

We employed a pre-test, training, post-test design. Infants participated in a free drumming measure of SMT pre and post experience of the Fast (138 BPM) or Slow (98 BPM) walking conditions. Caregivers were subsequently asked to complete additional parental measures.

Pre- and Post-Test Measure of Spontaneous Motor Tempo. Infants were seated on a cushion adjacent to the caregiver or on the caregiver's lap. A 12-inch drum supported on an adjustable height table was placed over the infant's lap. To familiarise the infant with the instrument, the experimenter demonstrated that the drum produces noise, telling the infant "Look!" and then hitting the drum once. If the infant did not spontaneously try to drum herself, the experimenter repeated the demonstration, leaving at least two seconds between each demonstration. In this way infants were not primed with a rate at which to hit the drum.

The trial started when the experimenter commenced the demonstration, and lasted for five minutes. Infants were congratulated when they hit or interacted with the drum. If infants moved away from the drum the caregiver returned the infant to their seated position. At the end of the trial infants were congratulated again, regardless of their behaviour during the trial.

Carrying experience - Fast condition. The caregiver placed the infant in a forward facing infant sling worn by the experimenter. The sling supported the infants weight so that the experimenter had both hands free. The experimenter, with infant, stepped onto the treadmill. A display monitor facing the infant from a distance of $50 \mathrm{~cm}$ was turned on and displayed an infant cartoon. The experimenter remained stationary for one minute, and infant and experimenter heart rate was recorded using surface electromyography (EMG). Following the heart rate recording, the experimenter started the treadmill and gradually increased the speed for up to one minute until it reached a comfortable speed at which to walk at 138 BPM (434 ms between steps). In order to keep pace whilst walking the experimenter listened to a metronome recording at 138 BPM through one in-ear headphone. The experimenter walked with both hands holding the handlebars and with easy access to the speed controls and emergency stop. The experimenter walked on the treadmill for 10 minutes. During the training, the caregiver was seated adjacent to the treadmill. Both experimenter and caregiver spoke to the infant in the first instance if the infant was not engaged with the video presented to them. An assistant also blew bubbles and provided toys if the infant became unsettled. At the end of the walking, the experimenter reduced the speed of the treadmill to a stop over the course of one minute, and then remained stationary on the treadmill for a further minute, whilst heart rate was again recorded. A digital camera recorded the training from the side such that the experimenter's feet were in shot throughout.

Carrying experience - Slow condition. The procedure for the Slow condition was identical to the Fast condition except that the treadmill speed facilitated walking at 98 BPM (612 ms between steps), and the experimenter could hear a corresponding metronome recording of 98 BPM.

Caregiver Measures. Following completion of infant testing, caregivers were asked to complete the following measures. Infants remained in the same room as the caregiver and were entertained by the experimenter's assistant.

Caregiver SMT measures. Caregivers took part in an abbreviated version of the infant drumming SMT task, where they were asked to sit within easy reach of the drum and drum consistently with one hand for one minute, with a smooth gesture, and at a comfortable, regular rate. They also took part in a tapping task, where they tapped the surface of the drum with their index finger, also for one minute and following the same instructions as when drumming.

Caregiver walking cadence measure. Caregivers were asked to step on to the stationary treadmill and were familiarized with the emergency stop. The caregiver then started the treadmill and the experimenter gradually increased and decreased the speed using a two-up two-down stair casing procedure (prompted: "Is this rate better, or worse, than before?"), until the caregiver reported that they were walking at their most comfortable pace. The caregivers walked at this pace for one minute. A video of the caregiver's footsteps was recorded in profile. Walking cadence was measured in this way as there was not space for naturalistic overground walking (indoor or outdoor), and priority for the study was given to our primary, infant, measures. However, cadence is known to vary by context (e.g. Oberg, Karsznia \& Oberg, 1993), and the stair casing procedure is unlikely to be as sensitive as 
a free-walking procedure. Such limitations are addressed in the discussion.

Questionnaire measure. Caregivers completed a questionnaire method detailing infants' gross motor milestones and the amount of time their infant typically spends in different gross motor activities, including being carried in a sling.

Infant and Caregiver anthropometric measurements. We took measurements of parent and infant height, leg length, arm length, and weight. The experimenter took all measurements. Height was measured from the top of the head to the floor. Arm length was calculated by adding measurements from the spine to the shoulder to measurements from the shoulder to the wrist. Leg length was measured from the hipbone protrusion to the ankle. Adults were measured in a standing position. Infant arm and leg lengths were measured when standing (if able to hold themselves in a standing position), lying supine on the floor, or whilst being held by the caregiver, and height was always measured whilst lying supine on the floor. To calculate infant weight the caregiver or experimenter stood on scales with and without the infant and the experimenter calculated the difference.

\section{Data Processing}

Measures of Spontaneous Motor Tempo. For infants, the ScreenFlow video recordings of the drumming sessions were used to identify periods of drumming and determine the corresponding time point in the original Audacity sound file. The experimenter hand marked the onset of each hit (as defined by the first peak in the sound stream, see Figure 1a for example). For each 'bout' of drumming (i.e. series of hits) the time stamp of each hit onset was recorded, along with how many hits were in the bout, and whether the bout was produced by one hand drumming, both hands drumming simultaneously, or both hands in an alternating sequence. If data were so noisy that the onset of the drum hit was not distinguishable (i.e. because of wire noise, very low amplitude hitting, etc.), they were discarded. Each bout of drumming was considered separately, with a pause of more than two seconds between hits considered a break in drumming. To best match the adult literature on unimanual tapping, the following analyses were performed on the rate of unimanual hits, or on the first hand to strike during bimanual hits, with alternating sequences excluded.

Matlab (MATLAB R2015b, The MathWorks Inc., MA, USA) was used to calculate the Inter-OnsetInterval (IOI). The mean IOI was calculated for each participant and taken as a measure of SMT. The relative standard deviation (RSD; also known as the coefficient of variation -- the ratio of the standard deviation to the mean, expressed as a percentage) of the IOI was also calculated for each participant and taken as a measure of regularity, i.e., a low relative standard deviation indicated more consistent drumming. To be included as a 'bout', infants had to perform four sequential hits with no more than a two second IOI between hits (Rocha et al., 2021). Infants who did not have at least one such 'bout' at pre and post-test were excluded from further analyses. As a very high RSD would indicate that the infant was drumming with very little regularity and therefore the IOI would not be a good measure of SMT, two visual outliers with an RSD of over $35 \%$ (Rocha et al., 2021) were further excluded from analysis of the intervention. This left a final sample of 27 infants with pre- and post-test data.

For caregiver drumming and caregiver tapping, the inbuilt Audacity 'Beat Finder' analysis tool was used to automatically detect and mark the onset of beats produced by the caregiver, by identifying each instance the signal went past a set decibel. This criterion was modified for each individual participant to account for individual variations in the strength of the hit/tap. The experimenter visually inspected the marked file and ensured all beats had been represented faithfully (see Figure 1b for example).

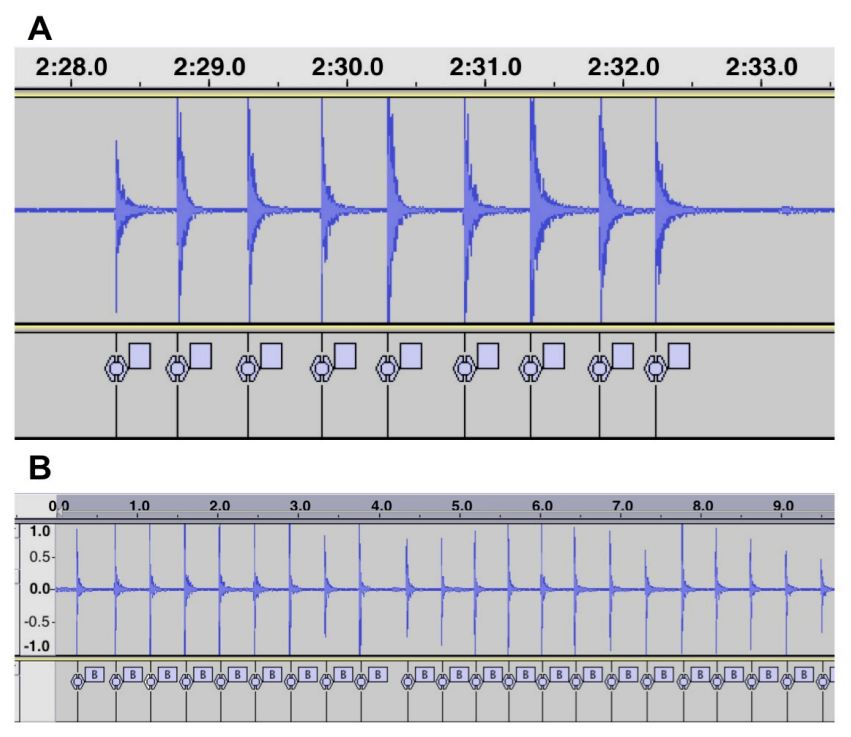

Figure 1a Example of manually event marked sound stream of infant drumming. Figure $1 \boldsymbol{b}$ Example of the event marking of an adult drumming, conducted with the Audacity ${ }^{\circledR}$ automatic beat finder function.

Caregiver Cadence. The number of steps that each caregiver took in one minute was coded from the 
profile view videotape. Steps per minute were translated to an Inter-Onset-Interval, giving milliseconds between steps as the independent variable, allowing easy comparison with the drumming and tapping data.

Heart rate. The infant EMG signal for the stationary period prior to walking and the stationary period immediately after walking were analysed using a custom built ProEMG pipeline, marking the onset of each heartbeat. Data where the heartbeat was not evident due to wire noise or signal dropout were discarded. This left pre- and post-test data for 20 infants; 10 in the Fast condition and 10 in the slow condition.

\section{Results}

Our primary hypothesis was that infant SMT would be influenced by experience of being carried at a novel rate; with infant SMT becoming faster from pre- to post-test if walked at the Fast speed of 138 BPM, and slower if walked at the Slow speed of 98 BPM. This was confirmed with a repeated measures ANOVA with infant SMT as the dependent variable, Time (PreTest or Post-Test) as a within subject factor and Condition (Fast or Slow) as a between subjects factor, revealing no main effects of Time $(\mathrm{F}(1,27)=.081, p=$ $.778)$ or Condition $(\mathrm{F}(1,27)=1.390, p=.249)$ but a significant Time*Condition interaction $(\mathrm{F}(1,27)=$ $\left.6.799, p=.015, \eta p^{2}=.201\right)$, such that infant SMT in the Fast condition became faster from pre- to post-test, and infant SMT in the Slow condition became slower from pre- to post-test (Fast Pre-Test $M=.495, S E=$ $.025,95 \%$ CI $(.445, .546)$; Fast Post-Test $M=.467$, $S E=.027,95 \%$ CI $(.412, .521)$; Slow Pre-Test $M=$ $.504, S E=.026,95 \%$ CI $(.452, .557)$; Slow Post-Test $M=.540, S E=.028,95 \%$ CI $(.484, .596)$; Figure 2A). One-tailed post-hoc paired t-tests confirm that the change in tempi were significant in both speed conditions $($ Fast $t(14)=1.99, p=.0335$; Slow $t(13)=$ $1.752, p=.052)$.

Much of the current literature on the impact of infant carrying has focused on the effect of vestibular stimulation on infant arousal, with studies showing a calming effect (Gordon \& Foss, 1966; Pederson \& Ter
Vrugt, 1973; Pederson, 1975; Hunziker \& Barr, 1986; Elliot et al., 1988; Esposito et al., 2013; Yilmaz \& Arikan, 2015), as has been physiologically documented by measuring respiration (Elliot et al., 1988) and heart rate (Esposito et al., 2013). We therefore further asked whether the differences in performance following the carrying experience could be explained by a differing state of arousal following the Fast walking compared to the Slow walking. If this were the case, we would expect an increase in heart rate where we see the faster drumming post-test in the Fast condition, and a decrease in heart rate in the Slow condition post-test. Figure 2B displays the mean heart rate IOI for infants in each condition.

Though these results are the opposite from what one would predict if change in infant SMT were driven by higher arousal, or more physical exertion (faster heartbeat) in the Fast condition, and lower arousal in the Slow condition, the change is not statistically significant. A repeated measures ANOVA with infant heart rate (IOI of heartbeats) as the dependent variable, Time (Pre-Test or Post-Test) as a within subject factor, and Condition (Fast or Slow) as a between subjects factor, confirms no significant effect of Time $(\mathrm{F}(1,18)=.612, p=.442)$ or Condition $(\mathrm{F}(1,18)=$ $.898, p=.356)$, and no evidence of a Time*Condition interaction $(\mathrm{F}(1,18)=1.585, p=.224)$.

In order to confirm that our data support the null hypothesis (no effect of carrying experience on infant heart rate), and is not the result of insufficient power, we used JASP (JASP Team 2017; Version 0.8.1.2) to calculate the Bayes Factors for the interaction, using the default priors. It is assumed that $\mathrm{BF}_{10}<.33$ provide good evidence to support the null (Jeffreys, 1939; Lee $\&$ Wagenmakers, 2014). The equivalent Bayesian repeated measures ANOVA revealed evidence for the null (Time*Condition $\mathrm{BF}_{10}=.263$ ), showing that there is over three times the evidence in our data that there is no effect of carrying rate on change in heart rate. We can therefore confirm that our SMT results are not explained by a change in arousal, as measured by heart rate. 
A

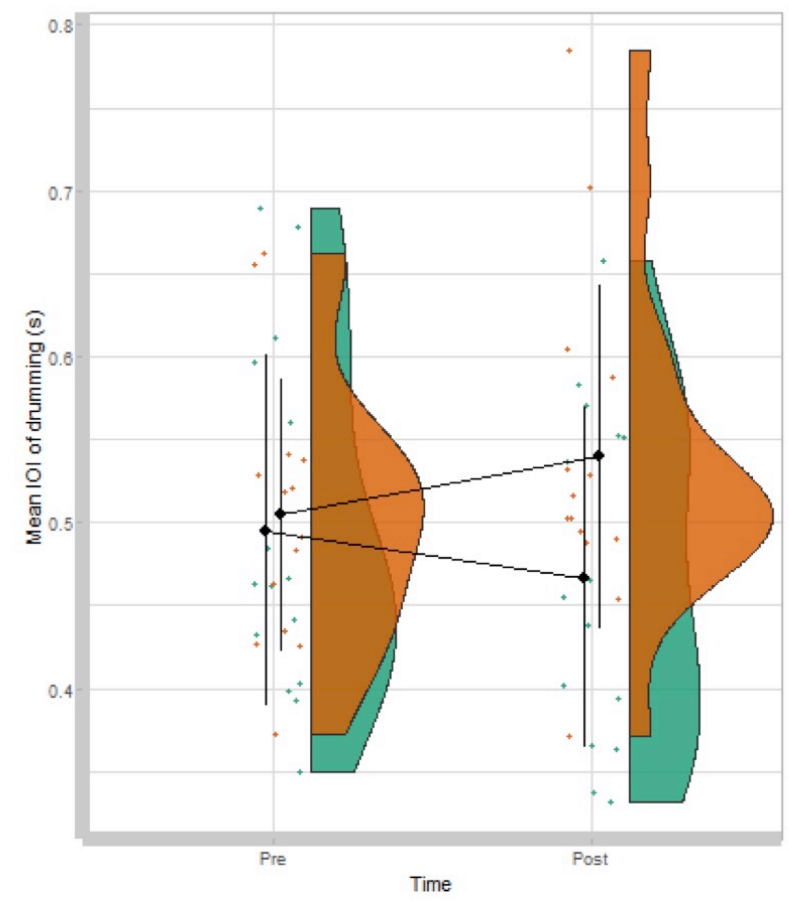

B

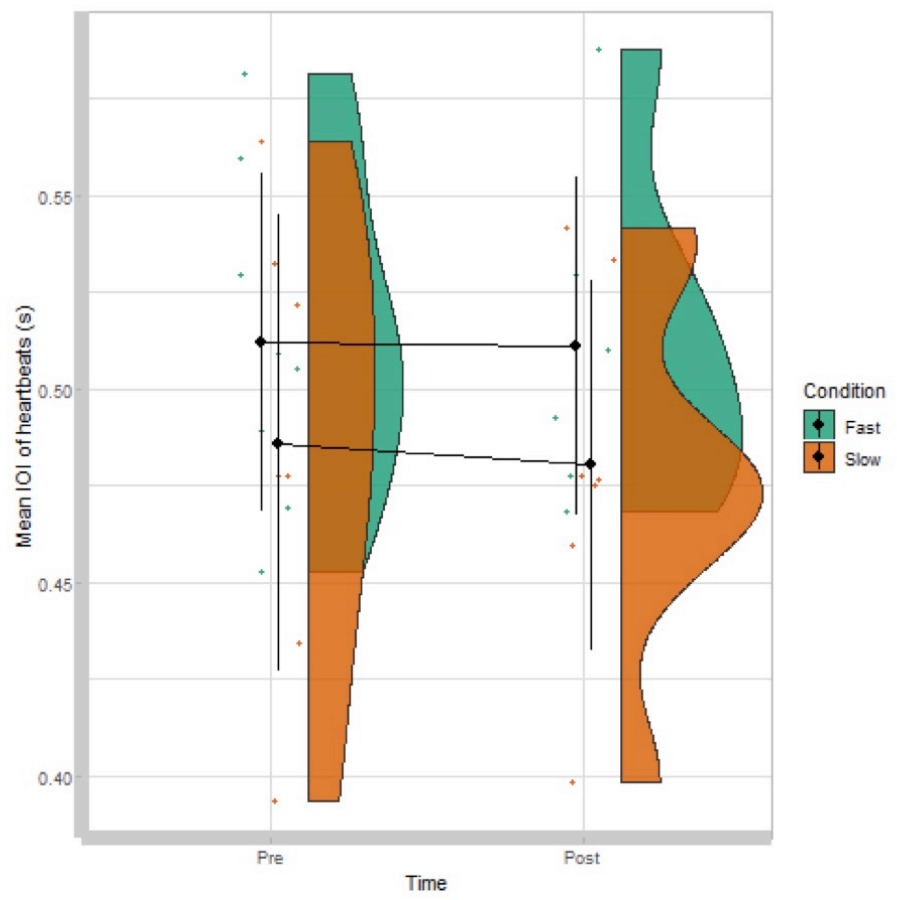

Figure 2a Graph to show interaction between Walking Condition (Fast or Slow) and Pre- and Post-Test measurement on infant SMT. Note: A faster SMT has a shorter ISI. Figure $2 \boldsymbol{b}$ Graph to show interaction between Walking Condition (Fast or Slow) and Pre- and Post-Test measurement on infant heart rate. Note: A faster heart rate has a shorter IOI.

\section{Parent measures}

In addition to our experimental manipulation, we were further interested in whether parents' rhythm as measured in the laboratory via drumming, tapping and crucially by walking cadence, would predict infant SMT. A linear regression with infant SMT at pre-test as the dependent variable and caregiver drumming rate, tapping rate, and stepping rate IOIs as predictors did not provide a significant model $(\mathrm{F}(3,28)=1.255, p=.309$, $\left.R^{2}=.119\right)$, and none of the predictors were significant (Parent Drum $\beta=.406, \mathrm{t}(28)=1.897, p=.068$; Parent Tap $\beta=-.173, \mathrm{t}(28)=-.805, p=.421$; Parent Walking Cadence $\beta=-.025, \mathrm{t}(28)=-.139, p=.891)$.

Similarly, linear regressions reveal that both infant and parent anthropometrics fail to predict infant SMT, and that parental anthropometrics fail to predict parent drumming and tapping. Table S1 displays the standardised coefficients, $t$ and $p$ values for all body measurements, both infant and parent, when predicting the dependent variable of infant pre-test SMT (infant measurements, $\mathrm{F}(4,30)=1.840, p=.147, R^{2}=.197$; parent measurements $\mathrm{F}(4,27)=.220, p=.925, R^{2}=$ $.032)$; and for parent measurements predicting parent drumming $\left(\mathrm{F}(4,36)=.613, p=.656, R^{2}=.064\right)$, and parent tapping $\left(\mathrm{F}(4,36)=1.491, p=.225, R^{2}=.142\right)$.

However, we do see a significant contribution of parent body size to parent walking cadence $(\mathrm{F}(4,30)=$ $3.832, p=.012, R^{2}=.338$ ). This is driven by a highly significant contribution of parent weight, such that heavier adults have a slower walking cadence than lighter adults (Parent Weight $\beta=.500, \mathrm{t}(30)=2.804, p$ $=.009)$. Parent weight is positively correlated with the other parent anthropometrics (all $p<.01$ ), and all parent anthropometrics are positively correlated with walking cadence, such that larger measurements correlate with slower walking, though only weight and arm length reach significance (for correlations see Table S2). As the anthropometrics were highly correlated, it is possible that overall stature is the best predictor of walking cadence. We therefore calculated a height*weight composite score ('Stature') and entered this as a predictor for parental cadence in a simple linear regression. The new Parent Stature variable predicts walking cadence $(\mathrm{F}(1,34)=10.092, p=.003$, $R^{2}=.229$ ). 
The current data fail to replicate that parental height predicts infant SMT (Rocha et al., 2021). This is likely due to the smaller sample size in the current study $(\mathrm{N}=32)$ than the former study $(\mathrm{N}=62)$. A Bayesian linear regression with the same factorial design as in Rocha et al. (2021) confirms that this model has inconclusive Bayes Factors (around 1) for all predictors (Parent Height BF10 =.621), showing that there is not evidence for an absence of an effect. Incorporating the 10-month-old data from the current study with the sample collected in Rocha et al. (2021), the previous finding of a significant model and parental height predicting infant SMT stands $((\mathrm{F}(6,85)=$ $3.576, p=.003, R^{2}=.202$; Parent Height $\beta=.364$, $\mathrm{t}(85)=2.509, p=.014)$.

\section{Infant motor activity}

Finally, we were interested in whether the rate or regularity of infant SMT was related to the types of experience of own locomotion and caregiver locomotion in which the infant participates, during her daily life. Two linear regressions with infant SMT and the RSD of infant SMT as dependent variables, and whether parents reported infants were carried for more than 30 minutes a day, could crawl or could cruise entered as predictors, revealed that although motor activity did not predict the rate at which infants drummed (infant SMT, $\left.\mathrm{F}(3,31)=.887, p=.459, R^{2}=.079\right)$, we find a significant model for the contribution of these activities to the variability in infant data (infant pre-test Relative Standard Deviation (RSD), F $(3,31)=4.037$ $\left.p=.016, R^{2}=.281\right)$, explaining $28 \%$ of the variance in the data. This result is driven by a highly significant effect of whether infants could cruise on infant RSD, such that infants who were cruising at the time of testing were more variable in their drumming (Cruising $\beta$ $=.532, \mathrm{t}(31)=3.349, p=.002)$. Full results are displayed in Table S3.

\section{Discussion}

We predicted that the experience of being carried at either a faster (138 BPM) or slower (98 BPM) than average walking pace would directly influence the SMT of 10 month old non-walking infants. Whilst performance between the two groups did not differ at pre-test, following ten minutes of being carried at a novel pace, infants in the fast group showed a faster SMT at posttest than infants in the slow group, with a mean rate of change across conditions of $20-30 \mathrm{~ms}$. We are thus the first to provide direct evidence that carrying infants can bias the rhythms that they spontaneous produce. Fur- ther, monitoring of infant heart rate before and after the walking training revealed no change in arousal from pre- to post-test, suggesting that the impact on SMT may be specific to rhythm, and unlikely to be the result of a general state change.

We took additional correlational measures of infant and parent body size, and parent rhythm, including parent cadence. Parental body size predicted parental walking cadence, partially corroborating the interpretation of Rocha et al. (2021), that parental body size predicts infant SMT as parent body size sets the tempo of parent walking. However, it was not the hypothesised height measurement that drove this result. Although the parental anthropometrics were all highly correlated, when measurements were entered into a multiple regression, weight was the strongest predictor, with arm length also marginally significant. That arm length was a predictor may reflect that it is a composite score, consisting of breadth (spine to shoulder) plus length (shoulder to wrist). When measuring cadence, indicators of overall stature may be more important than length measurements alone. Prior research on body size and walking cadence do show an impact of both weight and height, and both are normally used to standardise gait measurements (e.g. Pierrynowski \& Galea, 2001). This interpretation is supported by the significant predictive power of a composite Parent Stature measure. Whilst we fail to replicate the prior finding that parent height predicts infant SMT (Rocha et al., 2021), this is likely due to a lack of statistical power, as Bayesian analyses reveal that we are not finding evidence that support the null, but rather the data are inconclusive.

Whilst parent body size predicted parental cadence, we did not find an impact of parental cadence on parent's own SMT as measured by drumming or tapping, or on infant SMT as measured through drumming at pre-test. As such, we are unable to claim from our correlational measures that infant SMT is related to experience of locomotion at their caregiver's walking cadence. Our measure of caregiver cadence is a limitation of the study. Treadmill walking is known to be different to over-ground walking (e.g. Alton et al., 1998), and in our case, forced a non-continuous dependent variable, as there were limited speed options to walk at. For health and safety reasons, the caregiver was unable to carry their own child whilst on the treadmill, and caregiver motivation to be precise may have been low, especially if their infant was beginning to tire or fuss. That we do not find relationships within our adult measures may therefore reflect our suboptimal procedures (that were a direct consequence of our bespoke infant testing environment). Further, whilst the caregiv- 
er tested was the primary caregiver at time of the appointment, the recent introduction of equal maternity and paternity rights meant that we saw a mix of mothers and fathers. Though there was no difference to results if fathers were removed from the analyses reported, we did not collect specific data on time spent with different adults, and multiple caregiver families may weaken the effect we predicted to see. However, it is important to note that in the controlled laboratory environment our experimental manipulation worked, with novel experience changing infant SMT in the hypothesised directions, after only a ten-minute intervention. The limitations we note here can be rectified in future work by recording parents' natural behaviour, using accelerometers worn by the infant and caregiver. This kind of rich longitudinal time series data will be critical for a sensitive analysis exploring how the natural rhythms experienced in daily life impact infant SMT.

Finally, we find that infants who were cruising at the point of testing were more variable in their drumming than infants who were not cruising. Increased vestibular experience may benefit cognitive and motor functions (Korner \& Thoman, 1972; Rice, 1975; Gregg et al., 1976; Clark et al., 1977), likely through increased variability of experience (Dusing \& Hardbourne, 2010). Our findings are in line with Thelen's documentation of rigid, rhythmic stereotypies disappearing after the onset of more mature, volitional action; flexible and complex behaviour supersedes the regularity of initial motor outputs (Thelen, 1981). Whilst across the lifespan more variability in SMT may be viewed as a less mature rhythmic response, it is possible that in the first year of life, this less rigid performance may reflect the beginnings of greater motor control gained from the infants' own, diverse, locomotive experience.

Through a pre-test, training, post-test design, we successfully manipulated infant SMT with a ten-minute novel walking pace carrying intervention. This is the first direct evidence that carrying infants can change the rhythms that infants naturally produce. Our results suggest that an experience generally regarded as 'passive' on behalf of the infant may be shaping their earliest musical tendencies.

\section{References}

Alton, F., Baldey, L., Caplan, S., \& Morrissey, M. C. (1998). A kinematic

comparison of overground and treadmill walking. Clinical Biomechanics, 13(6), 434-440.

Ayres, B. (1973). Effects of infant carrying practices on rhythm in music. Ethos, 1(4), 387-404.
Bertram, J. E. (2005). Constrained optimization in human walking: cost minimization and gait plasticity. Journal of experimental biology, 208(6), 979-991.

Burger, B., Thompson, M. R., Luck, G., Saarikallio, S. H., \& Toiviainen, P. (2014). Hunting for the beat in the body: on period and phase locking in music-induced movement. Frontiers in Human Neuroscience, 8.

Clark, D. L., \& Chee, F. K. (1977). Vestibular stimulation influence on motor development in infants. Science, 196(4295), 1228-1229.

Dahl, S., Huron, D., Brod, G., \& Altenmüller, E. (2014). Preferred dance tempo: does sex or body morphology influence how we groove?. Journal of New Music Research, 43(2), 214-223.

Dienes, Z. (2014). Using Bayes to get the most out of nonsignificant results. Frontiers in psychology, 5, 781.

Dusing, S. C., \& Harbourne, R. T. (2010). Variability in postural control during infancy: implications for development, assessment, and intervention. Physical therapy, 90(12), 18381849

Elliott, M. R., Fisher, K., \& Ames, E. W. (1988). The effects of rocking on the state and respiration of normal and excessive cryers. Canadian Journal of Psychology/Revue canadienne de psychologie, 42(2), 163.

Esposito, G., Yoshida, S., Ohnishi, R., Tsuneoka, Y., del Carmen Rostagno, M., Yokota, S., ... \& Venuti, P. (2013). Infant calming responses during maternal carrying in humans and mice. Current Biology, 23(9), 739-745.

Esposito, G., Setoh, P., Yoshida, S. \&. Kuroda, K. "The calming effect of maternal carrying in different mammalian species." Frontiers in psychology 6 (2015).

Fraisse, P. (1982). "Rhythm and tempo," in The Psychology of Music, ed D.Deutsch (Orlando, FL: Academic Press), 149180.

Gordon, T., \& Foss, B. M. (1966). The role of stimulation in the delay of onset of crying in the newborn infant. The Quarterly Journal of Experimental Psychology, 18(1), 79-81.

Gregg, C. L., Haffner, M. E., \& Korner, A. F. (1976). The relative efficacy of vestibular-proprioceptive stimulation and the upright position in enhancing visual pursuit in neonates. Child Development, 309-314.

Hannon, E. E., Nave-Blodgett, J. E., \& Nave, K. M. (2018). The developmental origins of the perception and production of musical rhythm. Child Development Perspectives, 12(3), 194-198. 
Hunziker, U. A., \& Barr, R. G. (1986). Increased carrying reduces infant crying: a randomized controlled trial. Pediatrics, 77(5), 641-648.

Janata, P., Tomic, S. T., \& Haberman, J. M. (2012). Sensorimotor coupling in music and the psychology of the groove. Journal of Experimental Psychology: General, 141(1), 54.

Korner, A. F., \& Thoman, E. B. (1972). The relative efficacy of contact and vestibular-proprioceptive stimulation in soothing neonates. Child development, 443-453.

Lozoff, B., Brittenham, G. M., \& Klaus, M. (1978). Infant care-cache or carry?. Pediatric Research, 12, 373-373.

Mishima, J. (1965). Introduction to the morphology of human behavior. The experimental study of the mental tempo. Tokyo: Tokyo Publishing Co.

Pederson, D. R., \& Vrugt, D. T. (1973). The influence of amplitude and frequency of vestibular stimulation on the activity of two-month-old infants. Child Development, 122128.

Phillips-Silver, J., Trainor, L.J., (2005). Feeling the beat: movement influences infants' rhythm perception. Science, 308,1430 .

Phillips-Silver, J., \& Trainor, L. J. (2008). Vestibular influence on auditory metrical interpretation. Brain and cognition, 67(1), 94-102.

Pierrynowski, M. R., \& Galea, V. (2001). Enhancing the ability of gait analyses to differentiate between groups: scaling gait data to body size. Gait \& posture, 13(3), 193-201.

Provasi, J., Anderson, D. I., \& Barbu-Roth, M. (2014). Rhythm perception, production, and synchronization during the perinatal period. Frontiers in psychology, 5.

Provasi, J., \& Bobin-Bègue, A. (2003). Spontaneous motor tempo and rhythmical synchronisation in 2 $\frac{1}{2}$-and 4 -year-old children. International Journal of Behavioral Development, 27(3), 220-231.

Ravignani, A., Honing, H., \& Kotz, S. A. (2017). The evolution of rhythm cognition: Timing in music and speech. Frontiers in Human Neuroscience, 11.

Rice, R. D. (1975). Premature infants respond to sensory stimulation. APA Monitor, 6(11), 8-9.

Rocha, S., Southgate, V., \& Mareschal, D. (2020). Infant Spontaneous Motor Tempo. Developmental Science, e13032.
Styns, F., van Noorden, L., Moelants, D., \& Leman, M. (2007). Walking on music. Human movement science, 26(5), 769-785.

Teie, D. (2016). A comparative analysis of the universal elements of music and the fetal environment. Frontiers in psychology, 7, 1158.

Thelen, E. (1979). Rhythmical stereotypies in normal human infants. Animal behaviour, 27, 699-715.

Thelen, E. (1981). Rhythmical behavior in infancy: An ethological perspective. Developmental Psychology, 17(3), 237258.

Todd, N., Cousins, R., \& Lee, C. (2007). The contribution of anthropometric factors to individual differences in the perception of rhythm. Empirical Musicology Review, 2, 1-13.

McAngus Todd, N. P., O'Boyle, D. J., \& Lee, C. S. (1999). A sensory-motor theory of rhythm, time perception and beat induction. Journal of New Music Research, 28(1), 5-28.

Toiviainen, P., Luck, G., \& Thompson, M. R. (2010). Embodied meter: hierarchical eigenmodes in music-induced movement. Music Perception: An Interdisciplinary Journal, 28(1), 59-70.

Trainor, L. J. (2007). Do Preferred Beat Rate and Entrainment to the Beat Have a Common Origin in Movement? Empirical Musicology Review, 2, 17-20.

Trainor, L. J., Gao, X., Lei, J. J., Lehtovaara, K., \& Harris, L. R. (2009). The primal role of the vestibular system in determining musical rhythm. cortex, 45(1), 35-43.

Trevarthen, C. (2000). Musicality and the intrinsic motive pulse: evidence from human psychobiology and infant communication. Musicae Scientiae, 3(1 suppl), 155-215.

Wiernik, B. M. (2020, September 8). Preprint templates. https://doi.org/10.17605/OSF.IO/HSV6A

Winkler, I., Háden, G. P., Ladinig, O., Sziller, I., \& Honing, H. (2009). Newborn infants detect the beat in music. Proceedings of the National Academy of Sciences, 106(7), 24682471.

Yilmaz, G., \& Arikan, D. (2015). The effect of two different swinging methods upon colic and crying durations among the infants. Indian Journal of Pain, 29(3), 172. 
Table S1 Linear Regression Coefficients for effects of infant and parent anthropometrics on measures of infant and parent SMT

\begin{tabular}{lllllllllllll}
\hline & \multicolumn{1}{l}{ Infant Drum } & \multicolumn{3}{c}{ Parent Drum } & \multicolumn{3}{c}{ Parent Tap } & \multicolumn{3}{c}{ Parent Walk } \\
& $\boldsymbol{\beta}$ & $t$ & $p$ & $\boldsymbol{\beta}$ & $t$ & $p$ & $\boldsymbol{\beta}$ & $t$ & $p$ & $\boldsymbol{\beta}$ & $t$ & $p$ \\
\hline Infant Arm & .255 & 1.388 & .175 & $/$ & $/$ & $/$ & $/$ & $/$ & $/$ & $/$ & $/$ \\
Infant Leg & .142 & .752 & .458 & $/$ & $/$ & $/$ & $/$ & $/$ & $/$ & $/$ & $/$ & $/$ \\
Infant Height & .284 & 1.313 & .199 & $/$ & $/$ & $/$ & $/$ & $/$ & $/$ & $/$ & $/$ & $/$ \\
Infant Weight & -.137 & -.634 & .531 & $/$ & $/$ & $/$ & $/$ & $/$ & $/$ & $/$ & $/$ & $/$ \\
Parent Arm & -.133 & -.486 & .631 & -.086 & -.386 & .702 & .034 & .159 & .875 & .367 & 1.756 & .089 \\
Parent Leg & -.019 & -.060 & .953 & -.286 & -.990 & .329 & -.253 & -.916 & .366 & -.100 & -.337 & .738 \\
Parent Height & -.025 & -.083 & .935 & .137 & .483 & .632 & -.196 & .722 & .475 & -.244 & -.837 & .409 \\
Parent Weight & .097 & .426 & .674 & .157 & .824 & .415 & .268 & 1.466 & .151 & .500 & 2.804 & .009
\end{tabular}

Table $\boldsymbol{S} 2$ Correlations between parent anthropometrics

\begin{tabular}{|c|c|c|c|c|c|}
\hline & $\begin{array}{l}\text { Parent } \\
\text { Arm }\end{array}$ & $\begin{array}{l}\text { Parent } \\
\text { Leg }\end{array}$ & Parent Height & Parent Weight & Walking Cadence \\
\hline Parent & & $.642 * * *$ & $.581 * * *$ & $.454 * *$ & $.319^{\wedge}$ \\
\hline \multicolumn{6}{|l|}{ Arm } \\
\hline Parent & & & $.788 * * *$ & $.380 *$ & .137 \\
\hline \multicolumn{6}{|l|}{ Leg } \\
\hline Parent Height & & & & $.455 * *$ & .146 \\
\hline Parent Weight & & & & & $.478 * *$ \\
\hline
\end{tabular}

Table S3 Linear Regression Coefficients for effects of motoric experience on infant SMT and infant RSD

\begin{tabular}{lllllll}
\hline & Infant SMT & \multicolumn{3}{c}{ Infant RSD } \\
& $\boldsymbol{\beta}$ & $t$ & $p$ & $\boldsymbol{\beta}$ & $t$ & $p$ \\
Crawl & -.199 & -1.120 & .271 & -.231 & -1.472 & .151 \\
Cruise & .234 & 1.305 & .201 & .532 & 3.349 & .002 \\
Sling Use & -.053 & -.305 & .763 & -.013 & -.086 & .932
\end{tabular}

